\title{
El reenfoque en la selección de proveedores basado en la gestión de cadena de abastecimiento verde en empresa textil colombiana
}

Refocusing on supplier selection based on green supply chain management in Colombian textile company

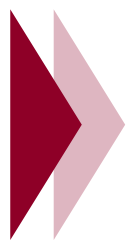

Pablo C. Ocampo

Estudiante del doctorado en Gestión Estratégica y Comercio Internacional, Universidad de Sevilla, España. Master in Management of Logistics Systems Ecole Polytechnique Federal de Lausanne Suiza.

E-Mail: pabloc.ocampo@ean.edu.co

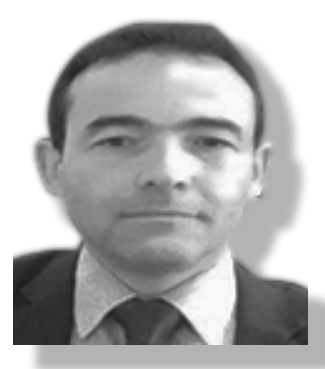

Ricardo Prada O

Ph.D. en Ciencias Empresariales por la Universidad Antonio de Nebrija, España; Doctor en Gestión por la Universidad EAN, Colombia; MBA-Magister en Administración; Especialista en Ingeniería de Producción; Ingeniero Mecánico y consultor empresarial. 


\section{RESUMEN}

La estrategia logística debe estar de la mano de un plan estratégico corporativo, el cual es de vital importancia para el desarrollo de la competitividad empresarial (Velásquez, 2003). Pero más allá de la logística, en la actualidad se está hablando de la cadena de abastecimiento, la gestión adecuada de cadena de abastecimiento la cual implica retos permanentes para las empresas debido a la globalización, es decir, la construcción de sinergias entre diferentes unidades de negocio y empresas, teniendo en cuenta que la logística es el trabajo para poner el inventario dentro de la red de valor y la cadena de suministro es la colaboración entre empresas que persiguen un mismo fin y posicionamiento estratégico común (Bowersox, Closs, \& Cooper, 2007).

Cada vez es más claro que si las cadenas de abastecimiento no se vuelven más sostenibles que lo que han sido en el pasado, los recursos del mundo y el medio ambiente no podrán mantener este nivel de crecimiento (Chopra \& Meindl, 2013, p. 501).

Con este artículo se pretende hacer una revisión del proceso de selección y evaluación de proveedores en una empresa del sector textil en Bogotá, con el cual se pretende contribuir a un reenfoque de la mejora del proceso actual, por medio de una propuesta de inclusión de criterios medioambientales basados en la investigación de diferentes autores especializados en gestión de cadena de abastecimiento verde.

Palabras claves: Cadena de abastecimiento, sector textil, competitividad, proveedores.

\section{ABSTRACT}

The logistics strategy must be the hand of a corporate strategic plan, which is vital for the development of business competitiveness (Velasquez, 2003). But beyond logistics, today is talking about the supply chain, the proper management of supply chain which involves permanent challenges for businesses due to globalization, which involves the construction of synergies between different business units and businesses, taking into account that logistics is working to put the inventory within the supply chain and supply chain is the collaboration between companies that have an objective and strategic positioning common (Bowersox, Closs, \& Cooper, 2007).

It is increasingly clear that if supply chains do not become more sustainable than they have been in the past, the world's resources and the environment cannot sustain this level of growth (Chopra \& Meindl, 2013, p. 501).

This article aims to review the selection process and evaluation of suppliers in a textile company in Bogotá, with which it is intended to contribute to the improvement of the current process, through a proposal for inclusion of environmental criteria based on research by different authors specializing in management green supply chain.

Keywords: Green Supply Chain Management, textile sector, competitiveness, suppliers.

\section{Introducción}

La revolución industrial creó los albores de lo que hoy constituye el mundo tecnológico moderno en el que vivimos. En la actualidad, el tema ambiental está vigente en un mundo en el que la población sigue creciendo a pesar de diferentes propuestas de control. En cuanto a la productividad y la prosperidad, la economía moderna está mostrando signos de errores y fallos de funcionamiento que no se previeron hace 100 años con inmensos daños al medio ambiente (Braungart, 2005, p. 23).

Hasta finales del siglo XX, una empresa podía ser muy exitosa sin tener sensibilidad por el medio ambiente (Wheelen \& Hunger, 2012). Por esto, cada vez existe más presión por parte de los interesados; ya no es posible estar en un mundo bajo las mismas condiciones bajo las que se creó la revolución industrial. Siendo la industria o la empresa cómplice y actor de tanto agotamiento de los recursos y hasta destrucción, es importante realizar investigación sobre estrategias para 
realizar mejoras en temas de sostenibilidad empresarial en pro de la sostenibilidad del medio ambiente.

De acuerdo con Bowersox, et al. (2007), la tendencia mundial está encaminada a la cadena de abastecimiento. En Colombia cuando se habla de logística se observa que generalmente, los empresarios no tienen claro cuáles son las implicaciones que este término tiene para el desarrollo de los procesos internos y externos de una organización (Sánchez, 2010).

No obstante, esta realidad empresarial observada mayoritariamente, se hacer relevante hablar de cadena de abastecimiento y su tendencia actual hacia una gestión de cadena de abastecimiento verde, con el fin de orientarse hacia una competitividad que no pase por alto el medio ambiente y preocuparse por el crear valores agregados para el cliente y sostenibilidad ambiental.

El presente artículo busca ofrecer una propuesta de oportunidad de mejora, realizando un reenfoque del proceso de selección y evaluación de proveedores, hacia la sostenibilidad de la cadena de abastecimiento de una empresa del sector textil (Comercializadora Textil Bogotái) y de confección en Colombia, mejora que podría impactar positivamente su enfoque hacia una cadena de suministro verde.

\section{Marco conceptual}

\subsection{La sostenibilidad}

Se refiere al uso de prácticas empresariales adecuadas para reducir el impacto ambiental (Wheelen \& Hunger, 2012, p. 9), por tanto, la sostenibilidad se ha convertido en una llave prioritaria en el diseño y la operación de las cadenas de suministro en el siglo XXI. Enfocarse en sostenibilidad permite a las cadenas de abastecimiento servir mejor a los clientes consientes del medio ambiente mientras mejoran su rendimiento de la cadena de abastecimiento (Chopra \& Meindl, 2013, p. 500).

1. Nombre ficticio, a solicitud de la dirección de la empresa textil investigada en Bogotá.
De este modo, la sostenibilidad ha presentado más de un desafío cuando requiere esfuerzos que no proveen retornos obvios en las inversiones para una compañía. De hecho, los mismos clientes no siempre han respaldado el tema de la sostenibilidad con una disposición a pagar más por productos sostenibles.

La sostenibilidad de la cadena de suministro puede ser medida en términos de:

- Consumo de energía

- Consumo de agua

- Emisión de gases de efecto invernadero

- Generación de residuos.

Es importante que estas medidas puedan ser seguidas a través de la cadena de abastecimiento con el mayor alcance posible (Chopra \& Meindl, 2013).

\subsection{Cadena de abastecimiento}

El concepto de la cadena de suministro y de todos sus componentes a partir de materias primas extraídas desde el primer proveedor hasta los productos de venta al por menor, listos para su compra por los consumidores (Council Supply Chain Management Professionals, 2016).

Adicional a lo anterior, el concepto de Cadena de Suministro: determinado como "un conjunto de tres o más entidades (organizaciones o individuos) que participan directamente en los flujos de aguas arriba y aguas abajo de los productos, servicios, finanzas, y / o información de una fuente a un cliente" (Mentzer, Dewitt, keebler, \& Zacharia, 2016).

Una cadena de suministro se compone de todas las partes involucradas, directa o indirectamente, para satisfacer la petición de un cliente (Sunil Chopra, 2013).

Según Marques, Paiva, Beheregarai, y Teixeira (2012), la cadena de suministro es un fenómeno que siempre se produce cuando las empresas establecen relaciones, independientemente del nivel de gestión existente. 
Según Christopher (1994), una cadena de suministro es "una la red de organizaciones que están involucradas, a través de aguas arriba y los enlaces descendentes, en los diferentes procesos $\mathrm{y}$ actividades que producen valor en forma de productos y servicios en las manos del cliente final”.

Una de las razones para el aumento del interés en el Supply Chain, es que las organizaciones se encuentran progresivamente dependientes de que tengan cadenas de suministro eficaces, o redes, para competir con éxito en el mercado global económico (Lambert 2008), lo cual requieren de una verdadera armonización de sus actores para un adecuado flujo de actividades.

Wei et al. (2007) expresa que la competitividad actual es entre cadenas de suministros y no entre empresas, lo cual es un pensamiento lógico (Naslund \& Williamson, 2010).

La cadena de suministro es dinámica y envuelve el flujo constante de información, producto y dinero entre los diferentes eslabones (Chopra \& Meindl, 2013).

\subsection{Gestión de la cadena de abastecimiento}

Es el resultado de la evolución natural de la gestión logística, cuyo quehacer se realiza silenciosa y cotidianamente, para lograr que los productos adecuados lleguen al lugar adecuado, en el instante adecuado, en condiciones adecuadas y al costo adecuado (Chaves \& Torres-Rabello, 2012, pág. 30).

Un análisis reciente de Stock et al. (2010) encontró que hay 166 únicas definiciones que hacen referencia "Supply Chain Management" en la literatura académica.

Desde su introducción como un concepto en la década de 1980, la gestión de la cadena de suministro (SCM) ha sido objeto de modificación y expansión significativa. Este autor identifica 3 temas principales asociados al Supply Chain y Supply Chain Management, donde se encuentra las (1) actividades; (2) beneficios; y (3) componentes (Stock, Boyer, \& Harmon, 2016).

\subsection{Gestión de la cadena de abastecimiento verde}

Este concepto, es determinado como una estrategia que vela por la óptima gestión ambiental dentro de las empresas, buscando un reenfoque en la manera de cómo se minimiza el impacto ambiental tales como la contaminación, el desperdicio de recursos y el producto vertido (Abu, 2012, p. 1)

\subsection{Selección de proveedores}

Acción de elegir a una persona o empresa que provee o abastece los recursos materiales (materias primas, equipos, herramientas, luz, agua, gas, etc.), recursos humanos (empleados y trabajadores que ofrecen sus servicios a cambio de una remuneración o salario) y recursos financieros necesarios para su operación diaria.

2. Análisis del entorno para la selección de proveedores en el sector textil con un enfoque de cadena de abastecimiento verde

La cadena de suministro del sector textil y confección está conformada por diferentes eslabones descritos en la Figura No. 1

Figura 1: Eslabones de la cadena de abastecimiento de sector textil y confección. Fuente: propia.

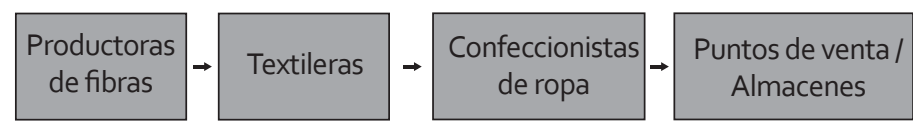

Para el caso de la empresa en estudio la red de cadena de abastecimiento está dada de acuerdo a la figura 2.

La Comercializadora Textil Bogotá realiza la compra de sus materias primas (telas), para la producción de sus prendas, estas materias prima son entregadas a sus plantas de confección quienes realizan todos los procesos industriales y de 
confección, para el despacho y entrega de las prendas de vestir para hombre a las 70 tiendas a nivel nacional y 5 tiendas internacionales.

El objetivo de la Comercializadora Textil Bogotá es tener los últimos diseños en las materias primas (telas) y el diseño más contemporáneo de las prendas de vestir para hombre. Todo lo anterior por las exigencias de los clientes finales que cada día necesitan tendencias y un gran portafolio de productos.

Figura 2: Red logística de la Comercializadora Textil Bogotá. Fuente: Comercializadora Textil Bogotá (2016).

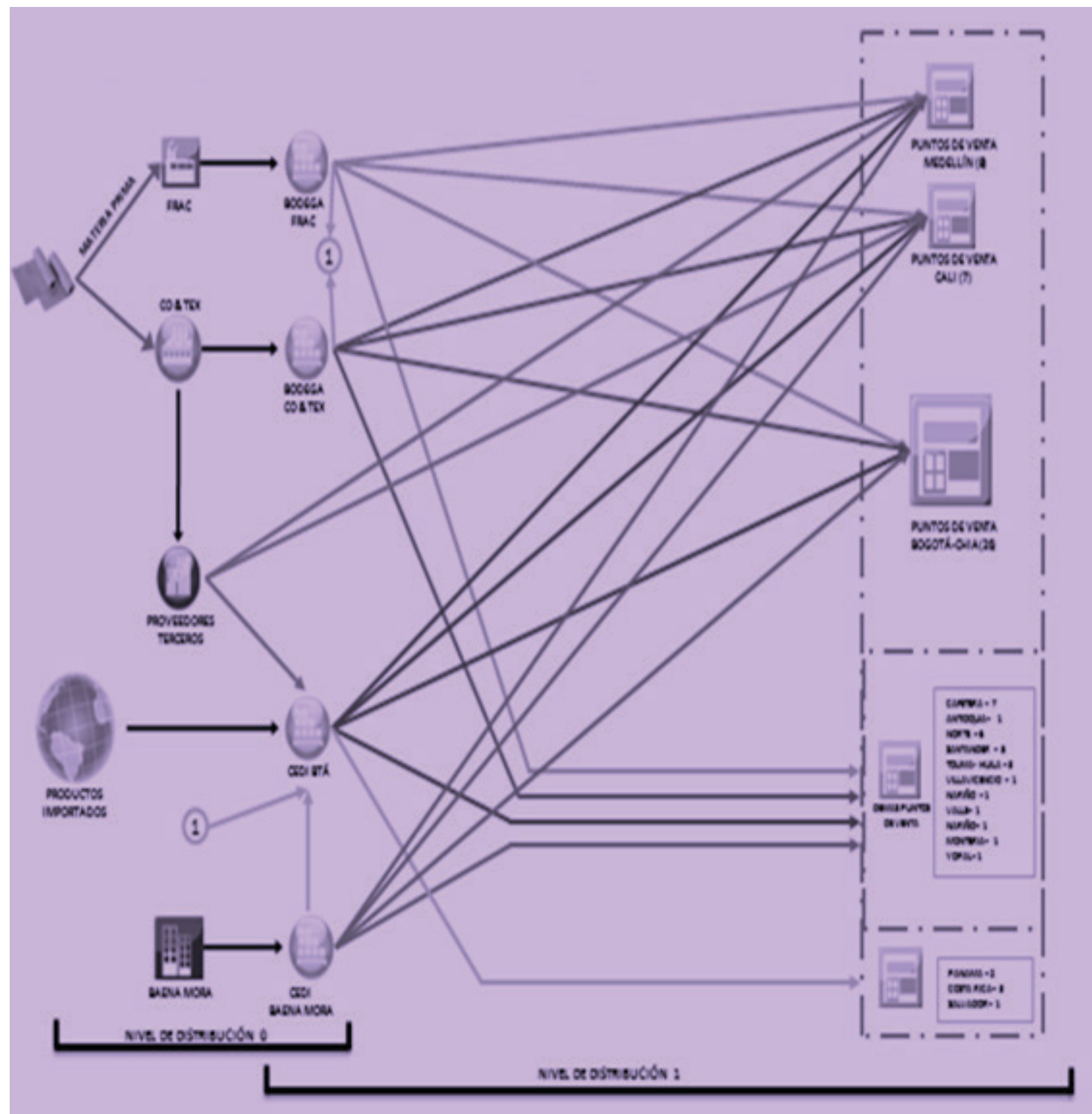


Revisando la red como punto de inicio es relevante la compra de materias primas llevado a cabo por la misma comercializadora, que como empresa líder de su red de suministro, tiene un alto poder de negociación con sus proveedores de materias primas, debido a su capacidad y volumen de compra. De esta manera este es un punto que se puede considerar clave, pues la empresa puede empezar a construir a través de la selección de sus proveedores un enfoque con base en la cadena de abastecimiento verde.

Al revisar el proceso actual de selección y evaluación de proveedores se evidenció que no son tenidos en cuenta criterios medio ambientales en la selección de estos. Por tal motivo se referencia este punto como una oportunidad de mejora para el proceso y enfocarlo así hacia una selección de proveedores más consiente en temas ambientales.

\section{Proceso de selección de proveedores para el aprovisionamiento estratégico medioambiental}

Como marco para el modelo de proveedores se ha tomado la información de Hanh, Rajagopal y Bernard (1993) quienes proponen un marco para la estrategia y el desarrollo del proveedor y Peterson propone un programa de compra ambiental. Este modelo resalta la importancia de tener una estrategia ambiental y una meta corporativa en la organización de compras, como también beneficios a largo plazo desde el proceso de selección de proveedores (Sarkis, 2010).

Este modelo propone que los involucrados en la compra sean capaces de entender la relación estratégica entre competitividad y proveedores. La necesidad de un programa ambiental debe ser reconocida desde la estrategia corporativa. En algunos casos la Dirección inicia el programa directamente.

Dentro del marco corporativo y las metas ambientales se deben tener cuenta medidas como el mejoramiento de la calidad, la reducción de costos y la reducción de desperdicios, la cual debe ser creada durante la fase de socialización con los proveedores. El proceso de recolección de información también debe incluir datos relevantes sobre evaluación y selección.
Según Frysinger (2001), los sistemas de decisión, soporte ambiental y los sistemas de direccionamiento ambiental empiezan hacer elementos críticos en el proceso de evaluación de la base de datos de suministro (Sarkis, 2010).

Las fuentes de información para estos datos no solamente provienen del mercado externo y de la competencia, resultan del programa de evaluación de proveedores, esta evaluación envuelve medidas de eficiencia, calidad, reducción de costos, entregas a tiempo e incluyen medidas específicas de prácticas ambientales de los proveedores como la certificación ISO 14000. Lo anterior implica programas de la prevención de la polución, reducción de desperdicios, gerenciamiento del desperdicio tóxico y la reunión de medidas de desempeño ambientales. Toda esta información debe tenerse en cuenta para tomar la decisión en la selección de proveedor. Si el proveedor no cumple con estos requisitos pero aún se considera dentro de la base de los proveedores, se puede implementar un programa de desarrollo de estos proveedores para el cumplimiento de estos requisitos.

La evaluación de proveedores es una parte integral del programa de desarrollo de los mismos. Los resultados sirven como guía para que el proveedor mejore sus resultados, o que por lo menos le preste más atención.

El siguiente paso para identificar las áreas para mejorar, y el propósito de esta fase es encontrar las causas específicas de los problemas. Los problemas de desempeño del proveedor pueden ser clasificados en capacidad técnica, manufactura, calidad, entrega y de gestión financiera. Esta clasificación ayuda a delimitar el área a ser investigada. En este punto del proceso los gerentes del proveedor deben ser invitados a participar en el análisis. El objetivo es obtener un diagnóstico y consenso involucrando al proveedor y al comprador, es muy importante el involucramiento temprano del proveedor en el análisis crítico para un éxito programa de implementación (Hanh, Rajagopal y Bernard, 1990).

Una vez identificadas las causas del problema, el equipo de implementación quien tiene la 
experiencia de organizar, diseña un plan y fija un cronograma con horarios, con el fin de determinar el grado de énfasis en cada área de desarrollo. Esto se realizará en conceso entre el comprador y el proveedor.

La parte final del programa de desarrollo del proveedor es la evaluación de resultados.

El consenso del plan de desarrollo debe ser implementado por el comprador y vendedor, cuando la implementación es completa los resultados son evaluados por objetivos generales y los objetivos específicos como son ambientales, técnicos, calidad, costos y capacidad de entrega. Es recomendado que este programe se trabaje adecuadamente en un corto tiempo, pero los proveedores participantes deben ser calificados y certificados por un largo plazo, los proveedores quienes no cumplen con este estatus deben ser eliminados de la base de datos de proveedores (Sarkis, 2010).

El programa de desarrollo de proveedores es una buena manera de medir el desempeño de los mismos, debido a que captura los atributos y resultados de los proveedores los cuales son necesarios medir, monitorear y gerenciar.

\section{Propuesta de mejora para la Comercializadora Textil Bogotá}

El proceso actual de Selección de proveedores en la Comercializadora Textil Bogotá utiliza los siguientes criterios:
a) Precio
b) Tiempo de entrega
c) Calidad
d) Garantía
e) Acuerdos de confidencialidad.

En la figura 2, se evidencia que no existen criterios relacionados enfocados en el sostenimiento medioambiental, por lo tanto se propone la inclusión de algunos criterios, que de no existir deben ser tenidos en cuenta e implementados por cada proveedor, de manera que se construya una base de proveedores para la sostenibilidad en la cadena de abastecimiento actual de la Comercializadora Textil Bogotá.
Para muchas firmas, la mayor parte de la energía, uso de agua y emisiones, ocurren en la extensión de su cadena de abastecimiento, afuera de su propio negocio. Por tanto, para tener un verdadero impacto en la sostenibilidad, los jugadores poderosos deben ver en la extensión de la cadena de suministro y trabajar con sus proveedores para mejorar el rendimiento (Chopra \& Meindl, 2013, p. 507).

De acuerdo a este planteamiento de Chopra \& Meindl (2013) en el que habla del papel de los jugadores fuertes en la cadena de abastecimiento, hace pensar en el liderazgo en la sostenibilidad de la cadena que pueden ejercer una empresa como la Comercializadora Textil Bogotá, en la que podría decirse que su impacto ambiental más relevante no está en su propio negocio de comercialización, si no a lo largo de su cadena. Un punto clave para iniciar el camino es trabajar con sus proveedores para el mejoramiento de la misma y disminuir el impacto negativo que pueden causar sus proveedores de telas, con relación al vertimiento de sustancias tóxicas a las fuentes de agua y el uso de químicos causantes de deterioro en la salud humana, estas medidas serán llevadas a cabo desde el proceso de selección de proveedores.

Basados en esta guía ambiental, se pueden proponer los siguientes criterios a ser tenidos en cuenta en el proceso de selección y evaluación de proveedores en la tabla 1:

Tabla 1: Criterios a tener en cuenta en selección de proveedores.

\begin{tabular}{|l|l|}
\hline Puntaje & $\begin{array}{l}\text { Criterio: Comportamiento Ambiental } \\
\text { Vertimientos } \\
\text { m3 de vertimientos / unidad de fibra }\end{array}$ \\
\hline 5 & $\begin{array}{l}\text { Ha implementado mejoras en reducción de } \\
\text { vertimientos }\end{array}$ \\
\hline 4 & $\begin{array}{l}\text { Lleva indicadores m3 de vertimientos / } \\
\text { unidad de fibra }\end{array}$ \\
\hline 3 & $\begin{array}{l}\text { Realiza mediciones, pero no evidencia planes } \\
\text { de acción respecto a estas }\end{array}$ \\
\hline 2 & $\begin{array}{l}\text { Esta en desarrollo de programas para } \\
\text { la implementación de mediciones de } \\
\text { vertimientos }\end{array}$ \\
\hline 1 & $\begin{array}{l}\text { No realiza medición o control sobre el } \\
\text { vertimiento }\end{array}$ \\
\hline
\end{tabular}




\begin{tabular}{|l|l|}
\hline Puntaje & Criterio: Normas ambientales \\
\hline 5 & Tiene sistema de gestión ambiental \\
\hline 4 & $\begin{array}{l}\text { Conoce y aplica indicadores para la gestión } \\
\text { ambiental }\end{array}$ \\
\hline 3 & $\begin{array}{l}\text { Tiene procedimiento para identificación de } \\
\text { normas }\end{array}$ \\
\hline 2 & $\begin{array}{l}\text { Evidencial el conocimiento de las normas } \\
\text { ambientales relacionadas con la empresa }\end{array}$ \\
\hline 1 & $\begin{array}{l}\text { No evidencia el conocimiento de las normas } \\
\text { ambientales relacionadas con la empresa }\end{array}$ \\
\hline
\end{tabular}

Fuente: Elaboración propia

\section{Metodología}

La investigación fue llevada a cabo con una metodología de revisión descriptiva, por medio de la búsqueda de referentes bibliográficos en las temáticas de proveedores globales y prácticas medioambientales, por medio de fuentes primarias y secundarias en el ámbito de proveedores verdes, y también se realizaron entrevistas semiestructuradas con líderes de proceso de la empresa textil. La revisión descriptiva proporciona al lector una puesta al día sobre conceptos útiles en áreas en constante evolución. Este tipo de revisiones tiene gran utilidad en la enseñanza y también interesará a muchas personas de campos conexos, porque leer buenas revisiones es la mejor forma de estar al día en nuestras esferas generales de interés (Day, 2005). La investigación se desarrolló siguiendo estos pasos: se definieron los objetivos de la revisión, se hizo la búsqueda bibliográfica o consulta de bases de datos y fuentes documentales o el establecimiento de la estrategia de búsqueda. Se especificaron los criterios de selección de documentos. Luego se hicieron entrevistas semiestructuradas a líderes de la empresa analizada, y finalmente, se organizó la información, para la redacción del artículo.

\section{Conclusiones}

Dada la importancia de los proveedores en la cadena de abastecimiento, se hace una propuesta de mejora en el proceso selección y evaluación de proveedores, con el fin de cumplir con un tema actualmente preponderante como lo es la responsabilidad ambiental en la sostenibilidad de dicha cadena.

Se considera que el proceso de obtención es de vital importancia para la Comercializadora textil Bogotá, puesto que es un factor diferencialy competitivo el cual impacta positivamente el CRM. Se desarrolló una propuesta para evaluar a los proveedores incluyendo unos criterios medioambientales y de comportamiento ambiental la cual se puede apreciar en la figura 4.

Figura 3: Propuesta para evaluar proveedores. Fuente: Propia.

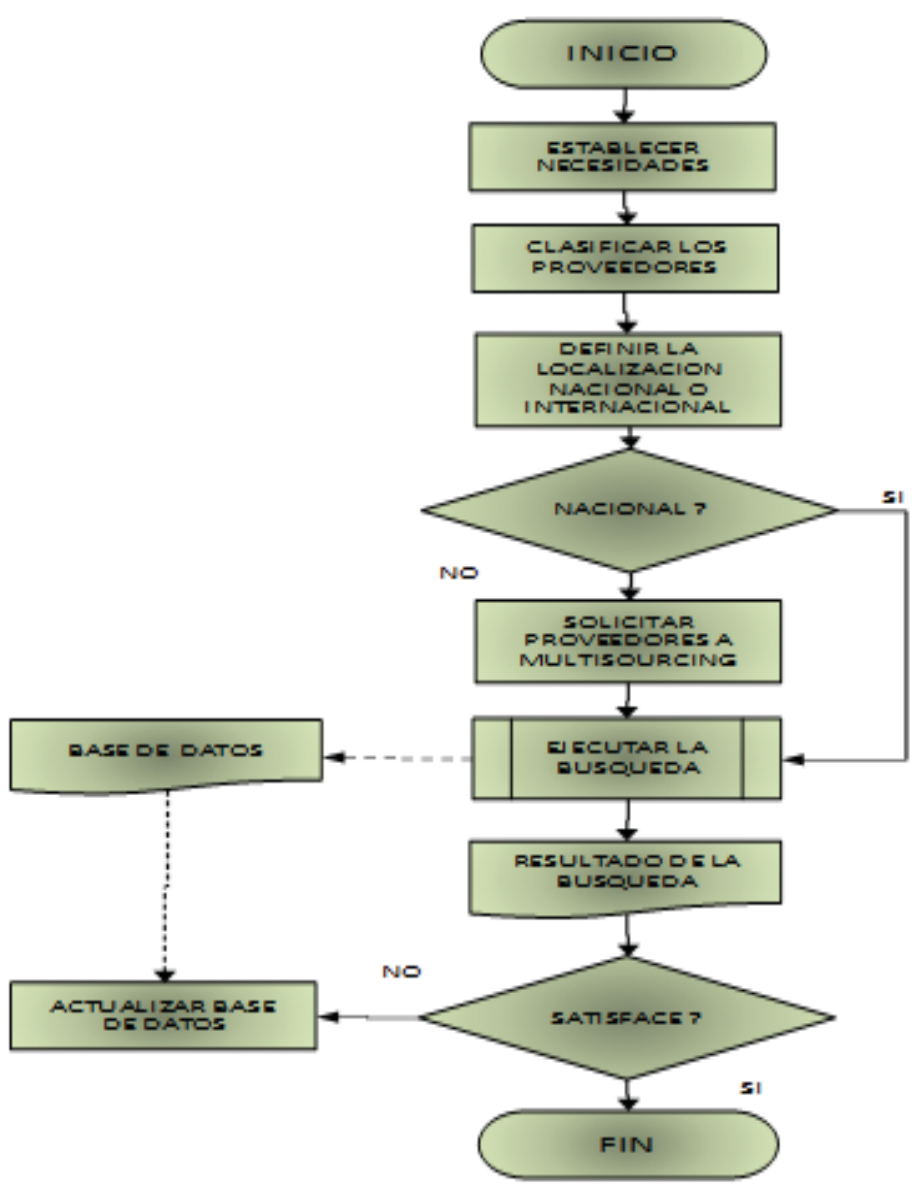


La importancia en la inclusión de estos criterios constituye la mejora y el desarrollo de los proveedores, de esta manera construimos cadenas colaborativas en donde los actores mejoren individualmente y creando sinergias hacia un futuro realmente sostenible.

Se considera que el proceso de compras es de vital importancia para la Comercializadora Textil Bogotá, puesto que es un factor diferencial y competitivo, el cual impacta positivamente el Customer Relationship Management. Además, para evaluar a los proveedores, se incluyen unos criterios medioambientales que cumplan con las tendencias de proveedores globales, los cuales se pueden apreciar en la Figura 2. La importancia de la inclusión de estos criterios constituye la mejora y el desarrollo de los proveedores globales, pues de esta manera construimos cadenas colaborativas en donde los actores mejoren individualmente $\mathrm{y}$ creen sinergias para un futuro realmente sostenible.

\section{REFERENCIAS}

Abu, N. A. (2012). The relationship of green supply chain management and green innovation concept. International Conference on Asia Pacific Business Innovation and, 5. Global. Décimo tercera edición. New Jersey: Pearson.

Arias, J. (2012). La gerencia verde. Bogotá: Universidad Sergio Arboleda.

Beltrán, N. (2014). Cadenas de abastecimiento verdes, oportunidades de negocio. Revista de Logistica. Recuperado de: http://www. revistadelogistica.com/cadenas-deabastecimiento-verdes-oportunidades-denegocio.asp.

Bowersox, D., Closs, D., \& Cooper, B. (2007). Administración y logística en la cadena de suministro. México: McGraw-Hill.

Braungart,M., McDonough W. (2005). Cradle to cradle: remaking the way we make things. Madrid: McGraw-Hill/Interamericana de España.
Comisión mundial sobre el medio ambiente y el desarrollo. (s.f.). Asamblea General de Naciones Unidas. Recuperado de http://www.un.org/ es/ga/president/65/issues/sustdev.shtml

Council Supply Chain Management Professionals. (21 de junio de 2016). Recuperado de: de Council Supply Chain Management Professionals: https://cscmp.org/education/ lincs/certification-tracks

Chaves, J., \& Torres, R. (2012). Supply chain management. Segunda edición. Santiago de Chile: RIL Editores.

Chopra, S. \& Meindl, (2013). Supply chain managment: strategy, planning, and operation. New Jersey: Pearson.

Garcia, L., Mora, A. (2008). Gestión logística integral. Bogotá: Ecoe Ediciones.

Hermida, M. (2008). La era de la logística verde. Latinpyme. 45: 46-47.

Mentzer, J. T., Dewitt, W., keebler, J., \& Zacharia, Z. G. (26 de Mayo de 2016). Researchgate. net. Recuperado de: Researchgate. net: https://www.researchgate.net/ publication/260386288_Defining_Supply_ Chain_Management.

Naslund , D., \& Williamson, S. (2010). What is Management in Supply Chain Management? - A Critical Review of. Journal of Management Policy and Practice vol. 11(4), 20.

Programa de transformación productiva. (2014). Recuperado de <https://www. ptp.com.co/contenido/contenido. aspx? conid $=910 \&$ catid $=643>$.

Sánchez R., Tomassian G. C. \& Pérez, G. (2010). Politicas integradas de infraestructura, transporte y logistica. México: Cepal.

Sarkis, J. (2010). Greening the supply chain. London: Sringer-Verlag London limited. 
Stock, J. R., Boyer, S. L., \& Harmon, T. (16 de junio de 2016). Research opportunities in supply chain management. Journal of the Academy of Marketing Science. 38 (1), 32-41. Recuperado de: http://link.springer.com/article/10.1007/ s11747-009-0136-2

Velásquez, A. (2003). Modelo de gestión de operaciones para pymes innovadoras. Revista Escuela de Administración de Negocios. 47, 6687.

Velásquez, A. (2005). Análisis situacional, intervención y aprendizaje organizacional. Revista Escuela de Administración de Negocios. 53, 52-71.

Wheelen, Thomas, L. \& Hunger J. (2012). Administración estrategica y politica de negocios. México: Pearson. 